\title{
Unusual finding during screening for intracardiac thrombus in patients referred for percutaneous left atrial appendage closure
}

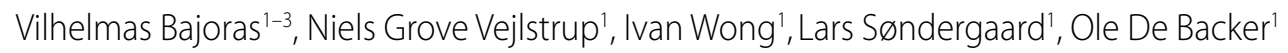 \\ 'The Heart Center, Rigshospitalet, Copenhagen University Hospital, Copenhagen, Denmark \\ ${ }^{2}$ Center of Cardiology and Angiology, Vilnius University Hospital Santaros Klinikos, Vilnius, Lithuania \\ ${ }^{3}$ Clinic of Cardiac and Vascular Diseases, Institute of Clinical Medicine, Faculty of Medicine, Vilnius University, Vilnius, Lithuania
}

\author{
Correspondence to: \\ Prof. Ole De Backer, MD \\ $\mathrm{PhD}, \mathrm{FESC}$, \\ The Heart Center, \\ Rigshospitalet - \\ University of Copenhagen, \\ Blegdamsvej 9, 2100 \\ Copenhagen, Denmark, \\ phone: +45 3545 7086, \\ e-mail: \\ ole.debacker@gmail.com \\ Copyright by the \\ Author(s), 2021 \\ Kardiol Pol. 2021; \\ 79 (6): 704-705: \\ DOI: 10.33963/KP.15958 \\ Received: \\ February 21, 2021 \\ Revision accepted: \\ April 11, 2021 \\ Published online: \\ April 16, 2021
}

A 65-year-old female, with a history of non-valvular atrial fibrillation and lower gastrointestinal bleeding while on oral anticoagulant therapy, was referred for percutaneous left atrial appendage (LAA) closure at the Heart Center, Rigshospitalet, Copenhagen University Hospital, Copenhagen, Denmark. Transthoracic echocardiography revealed a preserved left ventricular ejection fraction, mild mitral regurgitation, moderately enlarged left atrium and no intracardiac thrombi.

Pre-procedural cardiac computed tomography $(\mathrm{CT})$ showed an anatomically suitable LAA for catheter-based closure and excluded thrombus in the left atrium and LAA. Percutaneous LAA closure under local anesthesia with intracardiac echocardiography (ICE) guidance was, therefore, planned [1]. During the procedure, before transseptal puncture, ICE at the right ventricle position revealed there was a hypermobile, thrombus-like structure adhered to the calcified posterior mitral leaflet (Figure 1A-B; Supplementary material, Video S1-S2). Differential diagnosis of this intracardiac mass included thrombus or vegetation. Infective endocarditis was highly unlikely given negative blood culture and the absence of clinical signs of sepsis. Consequently, the procedure was interrupted, and a therapeutic dose of a low-molecular-weight heparin was initiated. Transesophageal echocardiography (TEE) was scheduled 6 weeks later to review the progression or resolution of the intracardiac mass. Retrospectively, this intracardiac mass adhered to the mitral valve could have been suspected at the pre-procedural cardiac CT scan (Figure 1C-D). In addition, other authors have previously reported routine TEE 24 hours before the LAA closure to exclude the presence of intracardiac thrombi [2].

Cardiac CT is increasingly adopted as the preferred imaging modality in the planning of catheter-based LAA closure [3, 4]. This report illustrates that screening for intracardiac thrombus should not only include the left atrium and LAA but all cardiac structures. Importantly, the essential role of intraprocedural echocardiography - either ICE or TEE - in the setting of percutaneous LAA closure should also be emphasized.

\section{Supplementary material}

Supplementary material is available at https:// journals.viamedica.pl/kardiologia_polska.

\section{Article information}

Conflict of interest: None declared.

Open access: This article is available in open access under Creative Common Attribution-Non-Commercial-No Derivatives 4.0 International (CC BY-NC-ND 4.0) license, allowing to download articles and share them with others as long as they credit the authors and the publisher, but without permission to change them in any way or use them commercially. For commercial use, please contact the journal office at kardiologiapolska@ptkardio.pl.

How to cite: Bajoras V, Vejlstrup NG, Wong I, et al. Unusual finding during screening for intracardiac thrombus in patients referred for percutaneous left atrial appendage closure. Kardiol Pol. 2021; 79(6): 704-705, doi: 10.33963/KP.15958. 


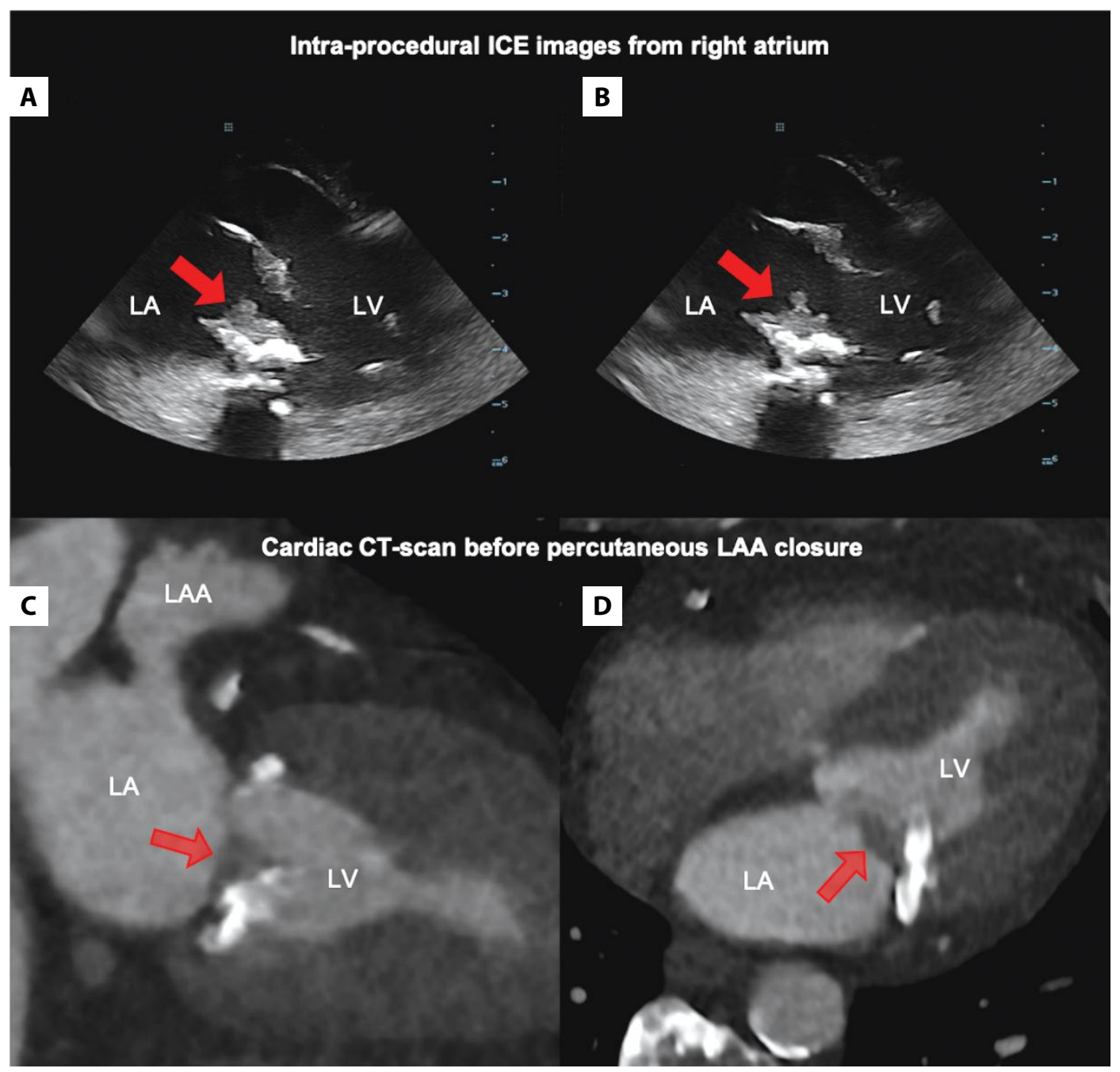

Figure 1. Intra-procedural intracardiac echocardiography and pre-procedural cardiac CT images. A, B. A thrombus-like structure (red arrow) shown by intra-procedural intracardiac echocardiography from the right atrium. C, D. Shady masses (red arrow) attached to the mitral valve as seen on the pre-procedural cardiac CT scan.

Abbreviations: $\mathrm{CT}$, computed tomography; ICE, intracardiac echocardiography; LA, left atrium; LAA, left atrial appendage; LV, left ventricular

\section{REFERENCES}

1. Nielsen-Kudsk JE, Berti S, De Backer O, et al. Use of intracardiac compared with transesophageal echocardiography for left atrial appendage occlusion in the amulet observational study. JACC Cardiovasc Interv. 2019; 12(11): 1030-1039, doi: 10.1016/j.jcin.2019.04.035, indexed in Pubmed: 31171278.

2. Burysz M, Litwinowicz R, Burysz A, et al. Causes of death and morbidity in patients with atrial fibrillation after left atrial appendage occlusion. Kardiol Pol. 2019; 77(11): 1047-1054, doi: 10.33963/KP.14966, indexed in Pubmed: 31495824.
3. Korsholm K, Berti S, Iriart X, et al. Expert recommendations on cardiac computed tomography for planning transcatheter left atrial appendage occlusion. JACC Cardiovasc Interv. 2020; 13(3): 277-292, doi: 10.1016/j. jcin.2019.08.054, indexed in Pubmed: 31678086.

4. de Backer O, Rosseel L, Søndergaard L. Are we too simple in planning complex structural interventions? The potential role of cardiac computed tomography to prepare for percutaneous left atrial appendage closure. Eurolntervention. 2019; 15(3): e213-e215, doi: 10.4244/EIJV15I3A38, indexed in Pubmed: 31186221. 